\title{
Plasma Exosome-Derived Fragile-Site Associated Tumor Suppressor is a Powerful Predictor of Prognosis in Patients with Ovarian Cancer
}

Renjing Hu ( $\square$ weiweihuhu112@163.com )

Wuxi Second People's Hospital

Xiaochun Chen

Taizhou Second People's Hospital

Shiliang Zhang

Wuxi Fifth People's Hospital

Bin Liu

Wuxi Fifth People's Hospital

Hao Pei

Wuxi Fifth People's Hospital

Fan Tu

Wuxi Fifth People's Hospital

Jun Liu

Wuxi Fifth People's Hospital

Hao Yu

Wuxi Fifth People's Hospital

\section{Research Article}

Keywords: fragile-site associated tumor suppressor (FATS), ovarian cancer, exosomal, prognosis, predictive value

Posted Date: June 29th, 2021

DOI: https://doi.org/10.21203/rs.3.rs-560273/v1

License: (c) (i) This work is licensed under a Creative Commons Attribution 4.0 International License. Read Full License 


\section{Abstract}

Objective. To investigate the levels of plasma exosome-derived fragile-site associated tumor suppressor (FATS) and evaluate its predictive ability in ovarian cancer (OC) patients.

Patients and Methods. Exosome-rich fractions were isolated from the plasma of enrolled 90 patients with OC. The levels of plasma exosome-derived FATS were detected with ELISA.

Results: The levels of exosome-derived FATS in OC patient were significantly lower than in healthy controls $(P<0.001)$. The levels of plasma exosome-derived FATS were obviously higher in OC patients with low grade (1/2), FIGO stages I/II than high grade (3/4), stages III/ IV disease $(P=0.003 ; P<0.001)$. The levels of plasma exosome-derived FATS were significantly higher in OC patients with no lymph node metastasis, no ascites than those with lymph node metastasis, ascites (both $P<0.001$ ). The levels of plasma exosome-derived FATS were obviously higher in OC patients with CA- 125 less than $35 \mathrm{U} / \mathrm{ml}$ than more than $35 \mathrm{U} / \mathrm{ml}(P<0.001)$. Among all enrolled OC patients, both 5-DFS and 5-OS were shorter in patients who had low plasma exosome-derived FATS levels than that high levels (both $P<0.001$ ). The AUROC of plasma exosome-derived FATS were $0.85(95 \% \mathrm{Cl}$ : 0.76-0.91) for 5-DFS, $0.91(95 \% \mathrm{Cl}: 0.83-0.96)$ for 5-OS prediction in patients with $\mathrm{OC}$, respectively.

Conclusions. Plasma exosome-derived FATS levels in OC patient were significantly down-regulated. Low levels of plasma exosome-derived FATS had close relationship with FIGO stages I/II, low grade, ascites, higher levels of CA-125, lymph node metastasis and prognosis of $\mathrm{OC}$ patients. Our findings may provide a new strategy in treating $\mathrm{OC}$.

\section{Introduction}

Ovarian cancer $(\mathrm{OC})$ is the gynecological malignant tumor with the highest fatality rate in the female reproductive system [1,2]. Its lack of obvious pathological features, difficulty in early diagnosis and lack of accurate tumor markers are all the reasons for poor prognosis of $\mathrm{OC}$ [3]. Nowadays, the incidence of $\mathrm{OC}$ is increasing worldwide, and the affected population is getting younger. There were more than 290,000 new cases of OC worldwide in 2018, with approximately 185,000 deaths [4,5]. At present, the main treatment methods are radical OC surgery, cytoreductive surgery, and platinum combined with paclitaxel chemotherapy, but the 5-year survival rate was still not ideal [6].

Oncology research includes the discovery of novel tumor suppressor genes and their role in the development of tumors [7]. Common fragile sites (CFS) are site-specific unstable regions in the normal genome, including rare fragile sites and CFS [8]. The relationship between rare fragile sites and tumors is not clear. However, CFS are consistent with many tumor genome mutation ones, and they are closely related to the occurrence and development of tumor [9]. The introns and expression regulatory regions of fragile-site associated tumor suppressor (FATS) gene are rich in AT repetitive sequences, with typical fragile locus gene characteristics [10]. FATS is a tumor suppressor gene associated with DNA damageinduced tumors, located at 10q26.2. Fluorescence in situ hybridization confirmed that FATS is located on 
the chromosome fragile site FRA10F. Because of the heterozygous loss of many tumors at this locus, the FATS gene may be closely related to the occurrence of human tumors [11]. Furthermore, it was found that the FATS gene not only has significant deletions in the DNA of breast cancer, OC, and lung cancer patients, but RT-PCR detection also showed that the expression levels of FATS mRNA were decreased or not expressed in $\mathrm{OC}$ cells and tumor tissues compared with the corresponding normal cells and adjacent tissues [12,13]. After co-transfecting the FATS expression vector into cells, it showed obvious anti-tumor activity in vitro and in vivo.

Exosomes are extracellular vesicles with a diameter of 30 to $150 \mathrm{~nm}$ secreted by various cells, Exosomes contains $m R N A$, proteins, lipids and other substances. Exosomes can also transmit information and substances between cells, and participate in the regulation of cell differentiation and tissue development $[14,15]$. Several studies have showed that there are four forms of information exchange between exosomes and cells: (1) Exosomes act as signal complexes to stimulate target cells; (2) Exosomes can transmit receptors between cells; (3) Exosomes can deliver functional proteins to recipient cells; (4) Exosomes can transmit genetic information to recipient cells. All above exchanges play a vital role in the occurrence, development, invasion and metastasis of tumors [16-18]. Recently, larger of studies have confirmed that exosomes can play an important role in the occurrence and development of OC by regulating the biological behavior of OC $[19,20]$. However, there are few reports on the diagnostic and prognostic value of exosomes in patients with OC. Hence, we wanted to investigate the levels of plasma exosome-derived FATS and assess its predictive ability in patients with OC.

\section{Materials And Methods}

\subsection{Patients}

The blood samples of 90 patients with OC were enrolled from the Second People's Hospital of Wuxi city, the Fifth People's Hospital of Wuxi city and the Second People's Hospital of Taizhou city between May 2010 to April 2016. Figure 1 showed a flow chart for enrolled patients with OC from the three hospitals. The following patients were excluded: (1) active infection; (2) coexisting hematologic malignancies, or other hematologic; (3) autoimmune disorders; (4) lack of clinical data; (5) follow up failed.

We collected the data of 90 patients with OC from medical records, including demographic characteristics, tumor size, lymph node metastasis, Federation International of Gynecology and Obstetrics (FIGO) stages and pathological differentiation. The median follow-up duration was 77.5 months (range: 48.0-112.0 months). During the same period, blood samples were collected from 50 healthy controls from the Wuxi Fifth People's Hospital, aged 51-73 years old, with the median age of 65 years old. The study had been approved by the hospital's Ethics Committee (No. 2017003), and the informed consent from each patient was obtained.

\subsection{Plasma exosome isolation}


Take out $1-2 \mathrm{ml}$ human plasma from the cells (dilute $1 \times$ PBS 5 times), and centrifuge at $500 \times \mathrm{g}$ for $5 \mathrm{~min}$ at $4^{\circ} \mathrm{C}$. After balancing, the supernatant was put into the centrifuge. The temperature of the centrifuge was set at $4{ }^{\circ} \mathrm{C}$ and centrifuged at $2000 \mathrm{~g}$ for $15 \mathrm{~min}$. then the supernatant was sub packed into a $15 \mathrm{ml}$ centrifuge tube and put into a $4{ }^{\circ} \mathrm{C}$ centrifuger. After balancing, the supernatant was collected by centrifugation at $15000 \mathrm{~g}$ for $1 \mathrm{~h}$, and then centrifuged for $0.22 \mu \mathrm{m}$. After aseptic filtration, continue to put the filter into a new ultracentrifugation tube. The temperature was set at $4{ }^{\circ} \mathrm{C}$ and centrifuged at 100000 $\mathrm{g}$ for $2 \mathrm{~h}$. After centrifugation, the supernatant was gently sucked away, leaving a precipitate. 100-200 $\mu \mathrm{l}$ $\times$ PBS buffer was used to resuspend the exosomes, which was left for storage at $-80^{\circ} \mathrm{C}$.

\subsection{Transmission electron microscopy (TEM)}

Prepare the extracted exosome from the $-80{ }^{\circ} \mathrm{C}$ refrigerator and thaw. The extracted exosomes were dissolved in $50-100 \mathrm{ml} \mu \mathrm{L} 2 \%$ paraformaldehyde solution, take $5-10 \mu \mathrm{l}$ exosome solution was added to the Formvar carbon sample carrier. Take $100 \mu$ PBS was added to the sealing film, and the copper mesh was placed on the PBS droplet for cleaning with tweezers. 50 $\mathrm{L} 1 \%$ glutaraldehyde dropped for 5 minutes, used $100 \mu \mathrm{LddH}_{2} \mathrm{O}$ to wash for 2 minutes (8 times). $50 \mathrm{ul}$ Uranium peroxide oxalate(ph7.0) dropped for $5 \mathrm{~min}$, 50ul Methylcellulose solution dropped for $10 \mathrm{~min}$. Put it on the stainless steel ring on the top of the sample table, absorb the excess liquid on the filter paper, and dry it in air for 5-10 minutes. Observe the shape of exosome under the electron microscope, take pictures and record.

\subsection{Nanoparticle tracking analysis (NTA)}

The sample was diluted with water and the particle concentration was $1 \times 10^{7} / \mathrm{ml}$ and $1 \times 10^{9} / \mathrm{ml}$. ZetaViewPMX110 instrument was used to measure the number and size of particles in the sample under $405 \mathrm{~nm}$ laser. Take 30 photos per second for $1 \mathrm{~min}$. According to the detection results, NTA software (ZetaView 8.02.28) was used to analyze the movement of particles and calculate the number of exosomes.

\subsection{Western blotting}

Dilute the extracted exosomes with RIPA lysate by a certain multiple, then draw the diluted Exo $20 \mu \mathrm{l}$, add it to the test sample well of a 96-well plate, and place it at room temperature. The total protein was obtained by separating exosomes and adding SDS buffer. After half dry transfer to PVDF film. Closed with $5 \%$ dried skimmed milk, and incubated overnight at $4{ }^{\circ} \mathrm{C}$ with the indicated primary antibodies (Annexin V, TSG101, CD9 and CD63) (Santa Cruz Biotechnology, Inc., Texas, USA). Shaked bed incubate at room temperature for 1 hour.

\subsection{ELISA}

Remove the residual cells from the plasma sample and dilute the cell debris with $1 \times$ PBS (1:500 dilution). On ice, the exosomes were precipitated with $100 \mathrm{ml}$ RIPA lysis buffer for half an hour. After shaking and mixing, PBS (1:3 dilution) was used to dilute the samples. Take out the ELISA plate coated with FATS antibody, and add 1 well of blank control and 7 wells of gradient concentration solution respectively. The 
diluted exosome-derived samples were $100 \mu \mathrm{l}$. After incubated at $37^{\circ} \mathrm{C}$ for $1 \mathrm{hour}$, discard the liquid in the well, spin dry then add $100 \mu \mathrm{l}$ of solution A, cover the membrane, and bath in a $37^{\circ} \mathrm{C}$ oven for 60 minutes, and wash the plate 3 times. Add $100 \mu \mathrm{l}$ of solution B, cover with membrane, incubate in the oven at $37{ }^{\circ} \mathrm{C}$ for $30 \mathrm{~min}$, wash the plate 5 times. $90 \mu \mathrm{l}$ of TMB substrate solution, cover with a film, and develop color in the dark at $37^{\circ} \mathrm{C}$ for $15 \mathrm{~min}$. Add $50 \mu \mathrm{l}$ of termination reaction solution. The microplate reader can detect the absorbance value at $450 \mathrm{~nm}$ wavelength.

\subsection{Statistical analysis}

Statistical analyses were performed with SPSS version 22.0 (IBM, Chicago, IL, USA). The data were shown as mean $\pm \mathrm{SD}$. Chi-square test or Wilcoxon's rank-sum test was used to perform correlation analyses. ROC curve analysis was used to assess the predictive ability of plasma exosome-derived FATS. $P$ value $<0.05$ was considered as significant.

\section{Results}

\subsection{Baseline characteristics}

The clinical characteristics of 90 enrolled OC patients are shown in Table 1. Among the enrolled OC patients, $49(54.44 \%)$ patients were less than 60 years of age, and $41(45.56 \%)$ patients were 60 years of age or older. The levels of CA-125 in 11 (12.22\%) patients were less than $35 \mathrm{U} / \mathrm{ml}$, while $79(87.78 \%)$ patients were more than $35 \mathrm{U} / \mathrm{ml}$. The pathological types included $52(57.78 \%)$ cases of ovarian serous carcinoma, $21(23.33 \%)$ cases of mucinous ovarian carcinom and $17(18.89 \%)$ cases of ovarian endometrioid carcinoma. 34(37.78\%) patients had FIGO stages I/II, while 56(62.22\%) patients had FIGO stages III/ IV disease. The numbers of patients with low grade (1/2), and high grade (3/4) were $43(47.78 \%)$, and $47(52.22 \%)$, respectively. Tumor size was less than $2 \mathrm{~cm}$ in 51 cases (56.67\%) and greater than $2 \mathrm{~cm}$ in 39 cases (43.33\%). Moreover, 53(58.89\%) patients had no lymph node metastasis, while $37(41.11 \%)$ patients had lymph node metastasis. 47 (52.22\%) patients showed ascites. In addition, the tumor position of $31(34.44 \%)$ patients, and $59(65.56 \%)$ patients were in one side and bilateral, respectively. 
Table 1

Baseline characteristics of enrolled OC patients

\begin{tabular}{|ll|}
\hline Characteristic & OC patients $(\mathbf{n}=90)$ \\
\hline Age(years) & \\
\hline$<60$ & $49(54.44)$ \\
\hline$\geq 60$ & $41(45.56)$ \\
\hline Tumor size(cm) & \\
\hline$<2$ & $51(56.67)$ \\
\hline$\geq 2$ & $39(43.33)$ \\
\hline Pathologic type & \\
\hline Ovarian serous carcinoma & $52(57.78)$ \\
\hline Mucinous ovarian carcinoma & $21(23.33)$ \\
\hline Ovarian endometrioid carcinoma & $17(18.89)$ \\
\hline Tumor grading & \\
\hline Low Grade (1/2) & $43(47.78)$ \\
\hline High Grade (3/4) & $47(52.22)$ \\
\hline Lymph node metastasis & \\
\hline YES & $37(34.44)$ \\
\hline NO & $37(41.11)$ \\
\hline FIGO stage & $53(58.89)$ \\
\hline I/II & \\
\hline III/ IV & \\
\hline CA-125(U/ml) & \\
\hline$<35$ & \\
\hline$\geq 35$ & \\
\hline Ascites & \\
\hline Negative & \\
\hline Positive & \\
\hline Position & \\
\hline
\end{tabular}




\begin{tabular}{|ll|}
\hline Characteristic & OC patients $(n=90)$ \\
\hline Bilateral & $59(65.56)$ \\
\hline
\end{tabular}

\subsection{Characteristics of exosomes}

The purification and exosome integrity were verified by TEM, NTA and western blot analysis. TEM images had a clear background and revealed that the single exosome diameter was between $100 \mathrm{~nm}$ and $200 \mathrm{~nm}$ and all the exosomes were clustered and connected. The shape had holonomic lipid capsule and double disc like vesicle structure (Fig. 2A). The NTA data showed that few number of particle diameter was between $0-50 \mathrm{~nm}$ and the main distribution was between 50 and $200 \mathrm{~nm}$, the median value of the total particles was approximately $100 \mathrm{~nm}$ (Fig. 2B). Western blot displayed that the expression of CD9, CD63, Tsg101, and Annexin $\vee$ were all positive (Fig. 2C).

\subsection{Correlation between plasma exosome-derived FATS levels and clinical pathological parameters}

We compared the levels of plasma exosome-derived FATS between OC patients and healthy controls, which showed that the exosome-derived FATS levels in OC patient were significantly down-regulated ( $P<$ 0.001 ; Fig. $3 \mathrm{~A}$ ). We also evaluated the levels of plasma exosome-derived FATS in OC patients with different disease stages. The levels of plasma exosome-derived FATS were significantly higher in OC patients with low grade (1/2) than high grade (3/4) $(P=0.003$; Fig. 3D). The levels of plasma exosomederived FATS were significantly higher in OC patients with no lymph node metastasis than those with lymph node metastasis $(P<0.001 ;$ Fig. 3E). The levels of plasma exosome-derived FATS were significantly higher in OC patients with FIGO stages I/II than stages III/ IV disease $(P<0.001$; Fig. 3F). The levels of plasma exosome-derived FATS were significantly higher in OC patients with CA-125 less than $35 \mathrm{U} / \mathrm{ml}$ than more than $35 \mathrm{U} / \mathrm{ml}(P<0.001 ;$ Fig. $3 \mathrm{H})$. The levels of plasma exosome-derived FATS were significantly higher in $\mathrm{OC}$ patients with no ascites than more than with ascites $(P<0.001$; Fig. 3I). However, there were no significantly statistical difference between patients with different age, tumor diameter and position (All $P>0.05$; Fig. 3B, 3C, 3G). Kaplan-Meier showed that both 5-OS and 5-DFS of $\mathrm{OC}$ patients with low levels of plasma exosome-derived FATS were shorter than those with high levels (both $P<0.001$; Fig. 4A, B).

\subsection{The predictive ability of plasma exosome-derived FATS for 5-year survival of patients with OC}

Next, we evaluated the predictive ability of exosome-derived FATS for 5-year survival of patients with OC (Table 2). The AUROC for 5-DFS was $0.85(95 \% \mathrm{Cl}$ : 0.76-0.91), with a sensitivity 73.9 (95\% Cl: $61.9-83.7$ ) and specificity $81.0(95 \% \mathrm{Cl}: 58.1-94.6)$. The cutoff value was 1277.41 . The positive predictive value and likelihood ratio were $92.7(95 \% \mathrm{Cl}: 82.4-98.0)$ and 3.88(95\% Cl: 1.6-9.5), respectively. The negative predictive value and likelihood ratio were $48.6(95 \% \mathrm{Cl}$ : $31.4-66.0)$ and $0.32(95 \% \mathrm{Cl}$ : $0.2-0.5)$, respectively. 
Table 2

The predictive ability of plasma exosome-derived

FATS for 5-year survival of patients with OC

\begin{tabular}{|ll|}
\hline Variable & OC patients $(\mathbf{n}=90)$ \\
\hline AUROC (5-DFS) & $0.85(0.76-0.91)$ \\
\hline Cutoff value (95\%Cl) & 1277.41 \\
\hline Sensitivity, \% & $73.9(61.9-83.7)$ \\
\hline Specificity, \% & $81.0(58.1-94.6)$ \\
\hline Positive predictive value, \% & $92.7(82.4-98.0)$ \\
\hline Negative predictive value, \% & $48.6(31.4-66.0)$ \\
\hline Positive likelihood ratio & $3.88(1.6-9.5)$ \\
\hline Negative likelihood ratio & $0.32(0.2-0.5)$ \\
\hline AUROC (5-OS) & $0.91(0.83-0.96)$ \\
\hline Cutoff value (95\%Cl) & 1188.24 \\
\hline Sensitivity, \% & $81.1(68.0-90.6)$ \\
\hline Specificity, \% & $91.9(78.1-98.3)$ \\
\hline Positive predictive value, \% & $93.5(82.1-98.6)$ \\
\hline Negative predictive value, \% & $77.3(62.2-88.5)$ \\
\hline Positive likelihood ratio & $10.01(3.4-29.8)$ \\
\hline Negative likelihood ratio & $0.21(0.1-0.4)$ \\
\hline O.91(95\% Cl: 0.83-0.96), with
\end{tabular}

The AUROC for 5-OS was $0.91(95 \% \mathrm{Cl}: 0.83-0.96)$, with the cutoff value of 1188.24 . The positive predictive value and likelihood ratio were 93.5(95\% Cl:82.1-98.6) and 10.01(95\% Cl:3.4-29.8), respectively, while the negative predictive value and likelihood ratio were $77.3(95 \% \mathrm{Cl}: 62.2-88.5)$ and $0.21(95 \% \mathrm{Cl}: 0.1-0.4)$, respectively. The sensitivity and specificity were $81.1(95 \% \mathrm{Cl}: 68.0-90.6)$ and 91.9(95\% Cl:78.1-98.3), respectively.

\section{Discussion}

Genomic instability is a common feature of tumor cells, which is characterized by abnormal DNA damage repair mechanism and disorder of cell cycle regulation [21]. The occurrence and development of tumor is the result of multi gene and multi-stage synergy, which is related to the genetic factors such as oncogene activation and tumor suppressor gene inactivation, and is affected by a variety of environmental factors [22]. DNA damage is an important mechanism in the occurrence and development of malignant tumors. Fragile sites of tumor suppressor genes on chromosomes are highly sensitive to DNA damage. The low 
expression or deletion of these genes may be highly correlated with the occurrence and development of tumor [23]. In recent years, there are more and more researches on fragile sites [24]. Fragile sites are an unstable region in normal genome, including 39 rare fragile sites and 88 common fragile sites. In normal cell culture, there was no chromosome fragile site. When DNA replication in cells is partially inhibited, a gap or a break region is formed in the metaphase of mitosis, which has been found in many cancers [25]. CFS locus genes are evolutionarily conserved, and are closely related to tumorigenesis and development. At the same time, they may also participate in the regulation of DNA damage response signaling pathways. It is a challenging frontier topic to find new ways to identify CFS locus genes, discover and identify new CFS genes, and clarify the relationship between CFS genes and carcinogenesis. Recent studies have found that FATS gene is very likely to be a candidate molecular marker that plays a key regulatory role in the early stage of tumorigenesis, and can be applied to the molecular diagnosis and risk prediction of cancer, which is of great significance for the study of tumorigenesis and development, and can provide new strategies for the prevention and treatment of cancers $[26,12,13]$.

In previous studies, Song et al. showed the role of FATS-p53 signaling cascade in inhibiting pregnancy related carcinogenesis and the potential application of FATS genotyping in breast cancer prevention [12]. Zhang et al found that the low expression of FATS was associated with breast cancer, and the expression of FATS had a good predictive value in DFS of breast cancer patients [13]. Wu et al. confirmed that the expression of FATS protein was related to the occurrence and development of non-small cell lung cancer (NSCLC), and was an independent prognostic factor of NSCLC. The detection of FATS protein was expected to become a new biomarker to evaluate the prognosis of patients with NSCLC [10]. However, the expression and biological role of FATS in $\mathrm{OC}$ are still unclear, especially in exosomes.

In this study, we extracted exosomes from the plasma of patients with OC. The purification and exosome integrity were verified by TEM, NTA and western blot analysis. TEM images had a clear background and revealed that the single exosome diameter was between $100 \mathrm{~nm}$ and $200 \mathrm{~nm}$ and all the exosomes were clustered and connected. The shape had holonomic lipid capsule and double disc like vesicle structure. The NTA data showed that few number of particle diameter was between 0-50 nm and the main distribution was between 50 and $200 \mathrm{~nm}$, the median value of the total particles was approximately 100 $\mathrm{nm}$. Western blot revealed that the expression of Annexin V, Tsg101, CD9 and CD63 were all positive for plasma exosomes.

We compared the levels of plasma exosome-derived FATS between OC patients and healthy controls. Compared with healthy controls, exosome-derived FATS levels in OC patient were significantly downregulated. Next, we evaluated the levels of plasma exosome-derived FATS in OC patients with different disease stages. The levels of plasma exosome-derived FATS were significantly higher in OC patients with low grade (1/2) than high grade (3/4). The levels of plasma exosome-derived FATS were significantly higher in $\mathrm{OC}$ patients with no lymph node metastasis than those with lymph node metastasis. The levels of plasma exosome-derived FATS were significantly higher in OC patients with FIGO stages I/II than stages III/ IV disease. The levels of plasma exosome-derived FATS were significantly higher in OC patients with CA-125 less than $35 \mathrm{U} / \mathrm{ml}$ than more than $35 \mathrm{U} / \mathrm{ml}$. The levels of plasma exosome-derived 
FATS were significantly higher in OC patients with no ascites than more than with ascites. However, there were no significantly statistical difference between patients with different age, tumor diameter and position. Among all enrolled OC patients, patients with low plasma exosome-derived FATS levels had both shorter 5-DFS and 5-OS.

We also evaluated the prognostic value of plasma exosome-derived FATS for predicting 5-year survival of patients with OC with ROC curve analysis. The AUROC of plasma exosome-derived FATS was $0.85(95 \%$ Cl: $0.76-0.91)$ for 5-DFS prediction in patients with OC, with a sensitivity $73.9(95 \% \mathrm{Cl}: 61.9-83.7)$ and a specificity $81.0(95 \% \mathrm{Cl}$ : 58.1-94.6). For OS prediction in OC patients, the AUROC of plasma exosomederived FATS was $0.91(95 \% \mathrm{Cl}: 0.83-0.96)$, with a sensitivity $81.1(95 \% \mathrm{Cl}: 68.0-90.6)$ and a specificity 91.9(95\% Cl:78.1-98.3). Plasma exosome-derived FATS levels had good performances both in 5-OS and 5 -DFS of OC patients.

Our study also had some limitations. Firstly, although this was a larger study evaluating plasma exosome-derived FATS levels in OC patients, more patients from multiple centers need to be enrolled for validation. Secondly, our study did not assess the levels of plasma exosome-derived FATS in OC patients with different pathological types. Thirdly, we did not investigate the mechanism of exosome-derived FATS in occurrence and development of OC.

In summary, our study showed that plasma exosome-derived FATS levels in OC patient were significantly down-regulated. Low plasma exosome-derived FATS levels were closely related to the FIGO stages I/II, low grade, ascites, higher levels of CA-125, lymph node metastasis and prognosis of OC patients. These findings may facilitate the establishment of plasma exosome-derived FATS levels as a novel biomarker for prognosis in OC.

\section{Declarations}

Consent for publication: The authors confirmed that all specimens have obtained written consent from each patient.

Data availability: All data in this study are included in the manuscript.

Funding sources: This work was supported by the Youth Medical Talent of Jiangsu Province (grant no. QNRC2016163).

Authors' contributions: R.H directed and supervised the study and revised the manuscript. X.C. and S.Z. designed and performed most of the experiments. B.L. and H.P participated in some experiments, analyzed the data and completed the figures. F.T., J.L., and H.Y. wrote the manuscript. All authors read and approved the final manuscript.

Competing interests: All authors have declared that there is no competing interests exists.

\section{Abbreviations}


FATS: fragile-site associated tumor suppressor;

OC: ovarian cancer;

NTA: Nanoparticle tracking analysis;

TEM: Transmission electron microscopy;

5-DFS: 5-year disease-free survival;

5-OS: 5-year overall survival

\section{References}

[1] Menon Usha, Gentry-Maharaj Aleksandra, Burnell Matthew et al. Ovarian cancer population screening and mortality after long-term follow-up in the UK Collaborative Trial of Ovarian Cancer Screening (UKCTOCS): a randomised controlled trial. Lancet, 2021, doi:10.1016/S0140-6736(21)00731-5

[2] Kim Soyoun Rachel, Tone Alicia, Kim Raymond $\mathrm{H}$ et al. Maximizing cancer prevention through genetic navigation for Lynch syndrome detection in women with newly diagnosed endometrial and nonserous/nonmucinous epithelial ovarian cancer. Cancer, 2021, doi:10.1002/cncr.33625

[3] Wu J, Shang AQ, Lu WY. Clinical significance of NGAL and MMP-9 protein expression in epithelial ovarian cancers. Int J Clin Exp Med, 2016;9(2):3069-3075

[4] Dafni U, Martín-Lluesma S, Balint K et al. Efficacy of cancer vaccines in selected gynaecological breast and ovarian cancers: A 20-year systematic review and meta-analysis. Eur J Cancer, 2021, 142: 63-82.

[5] Lallemand Christophe, Ferrando-Miguel Rosa, Auer Michael et al. Quantification of Bevacizumab Activity Following Treatment of Patients With Ovarian Cancer or Glioblastoma. Front Immunol, 2020, 11: 515556.

[6] Odunsi Adekunle, McGray A J Robert, Miliotto Anthony et al. Fidelity of human ovarian cancer patientderived xenografts in a partially humanized mouse model for preclinical testing of immunotherapies. $J$ Immunother Cancer, 2020, 8: doi:10.1136/jitc-2020-001237

[7] Wang Yuan, Wu Jian, Xu Jiangyan et al. Clinical significance of high expression of stanniocalcin-2 in hepatocellular carcinoma. Biosci Rep, 2019, 39(4), doi:10.1042/BSR20182057

[8] Kołat Damian, Kałuzińska Żaneta, Płuciennik Elżbieta, WWOXFragile Gene Guides /-Dependent Actions Against Tumor Progression in Grade II Bladder Cancer. Front Oncol, 2021, 11: 621060.

[9] Mendelaar Pauline A J, Smid Marcel, van Riet Job et al. Whole genome sequencing of metastatic colorectal cancer reveals prior treatment effects and specific metastasis features. Nat Commun, 2021, 12: 574. 
[10] Qiu Li, Hu Linfei, Wang Huijuan et al. FATS regulates polyamine biosynthesis by promoting ODC degradation in an ERß-dependent manner in non-small-cell lung cancer. Cell Death Dis, 2020, 11: 839.

[11] Ma Ke, Qiu Li, Mrasek Kristin et al. Common fragile sites: genomic hotspots of DNA damage and carcinogenesis. Int J Mol Sci, 2012, 13: 11974-99.

[12] Song Fangfang, Zhang Jun, Qiu Li et al. A functional genetic variant in fragile-site gene FATS modulates the risk of breast cancer in triparous women. BMC Cancer, 2015, 15: 559.

[13] Zhang Jun, Gu Lin, Zhao Lu-Jun et al. Expression level of novel tumor suppressor gene FATS is associated with the outcome of node positive breast cancer. Chin Med J (Engl), 2011, 124: 2894-8.

[14] Su Dandan, Tsai Hsiang-I, Xu Zhanxue et al. Exosomal PD-L1 functions as an immunosuppressant to promote wound healing. J Extracell Vesicles, 2019, 9: 1709262.

[15] Jarvis Rachel, Tamashiro-Orrego Alessandra, Promes Vanessa et al. Cocaine Self-administration and Extinction Inversely Alter Neuron to Glia Exosomal Dynamics in the Nucleus Accumbens. Front Cell Neurosci, 2019, 13: 581.

[16] Adem Bárbara,Vieira Patricia F, Melo Sonia A, Decoding the Biology of Exosomes in Metastasis. Trends Cancer, 2020, 6: 20-30.

[17] Petanidis Savvas, Domvri Kalliopi, Porpodis Konstantinos et al. Inhibition of kras-derived exosomes downregulates immunosuppressive BACH2/GATA-3 expression via RIP-3 dependent necroptosis and miR146/miR-210 modulation. Biomed Pharmacother, 2020, 122: 109461.

[18] Wang Li, Wu Jian, Song Shu et al. Plasma Exosome-Derived Sentrin SUMO-Specific Protease 1: A Prognostic Biomarker in Patients With Osteosarcoma. Front Oncol, 2021, 11: 625109.

[19] Liu Jingjing, Yoo Jigeun, Ho Jung Yoon et al. Plasma-derived exosomal miR-4732-5p is a promising noninvasive diagnostic biomarker for epithelial ovarian cancer. J Ovarian Res, 2021, 14: 59.

[20] Ma Ruiqiong, Ye Xue, Cheng Hongyan et al. Tumor-derived exosomal circRNA051239 promotes proliferation and migration of epithelial ovarian cancer. Am J Trans/ Res, 2021, 13: 1125-1139.

[21] Ullah Asmat, Leong Sze Wei, Wang Jingjing et al. Cephalomannine inhibits hypoxia-induced cellular function via the suppression of APEX1/HIF-1a interaction in lung cancer. Cell Death Dis, 2021, 12: 490.

[22] Dong Baijun, Fan Liancheng, Yang Bin et al. Use of Circulating Tumor DNA for the Clinical Management of Metastatic Castration-Resistant Prostate Cancer: A Multicenter, Real-World Study. J Natl Compr Canc Netw, 2021, doi:10.6004/jnccn.2020.7663

[23] Brahme Anders,Hultén Maj,Bengtsson Carin et al. Radiation-Induced Chromosomal Breaks may be DNA Repair Fragile Sites with Larger-scale Correlations to Eight Double-Strand-Break Related Data Sets 
over the Human Genome. Radiat Res, 2019, 192: 562-576.

[24] Pladevall-Morera David,Munk Stephanie,Ingham Andreas et al. Proteomic characterization of chromosomal common fragile site (CFS)-associated proteins uncovers ATRX as a regulator of CFS stability. Nucleic Acids Res, 2019, 47: 8004-8018.

[25] Wang Hailong,Li Shibo,Zhang Huimin et al. BLM prevents instability of structure-forming DNA sequences at common fragile sites. PLoS Genet, 2018, 14: e1007816.

[26] Zhang Jun,Wu Nan,Zhang Tiemei et al. The value of FATS expression in predicting sensitivity to radiotherapy in breast cancer. Oncotarget, 2017, 8: 38491-38500.

Figures

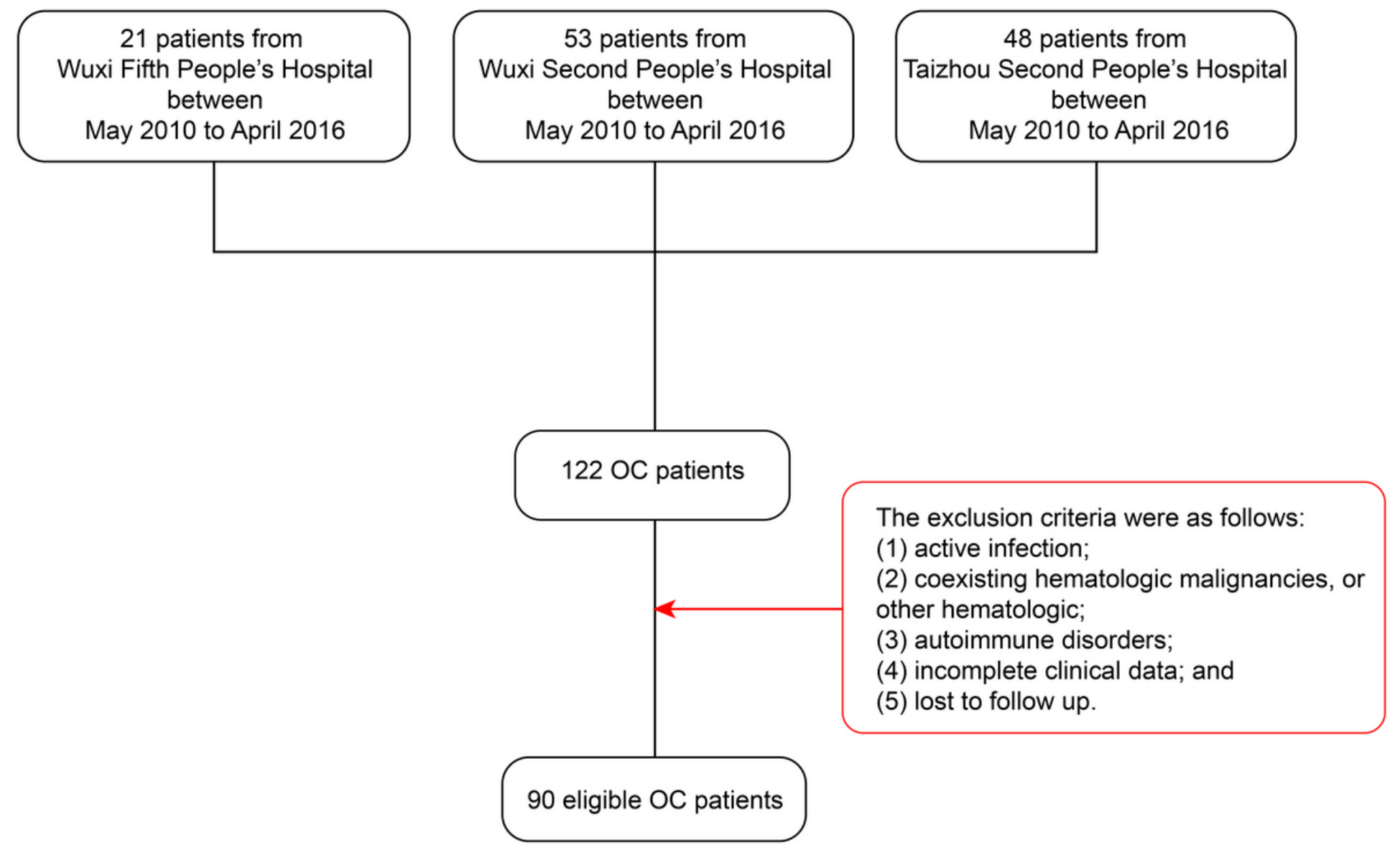

\section{Figure 1}

The flow chart for enrolled patients with OC from the three hospitals 

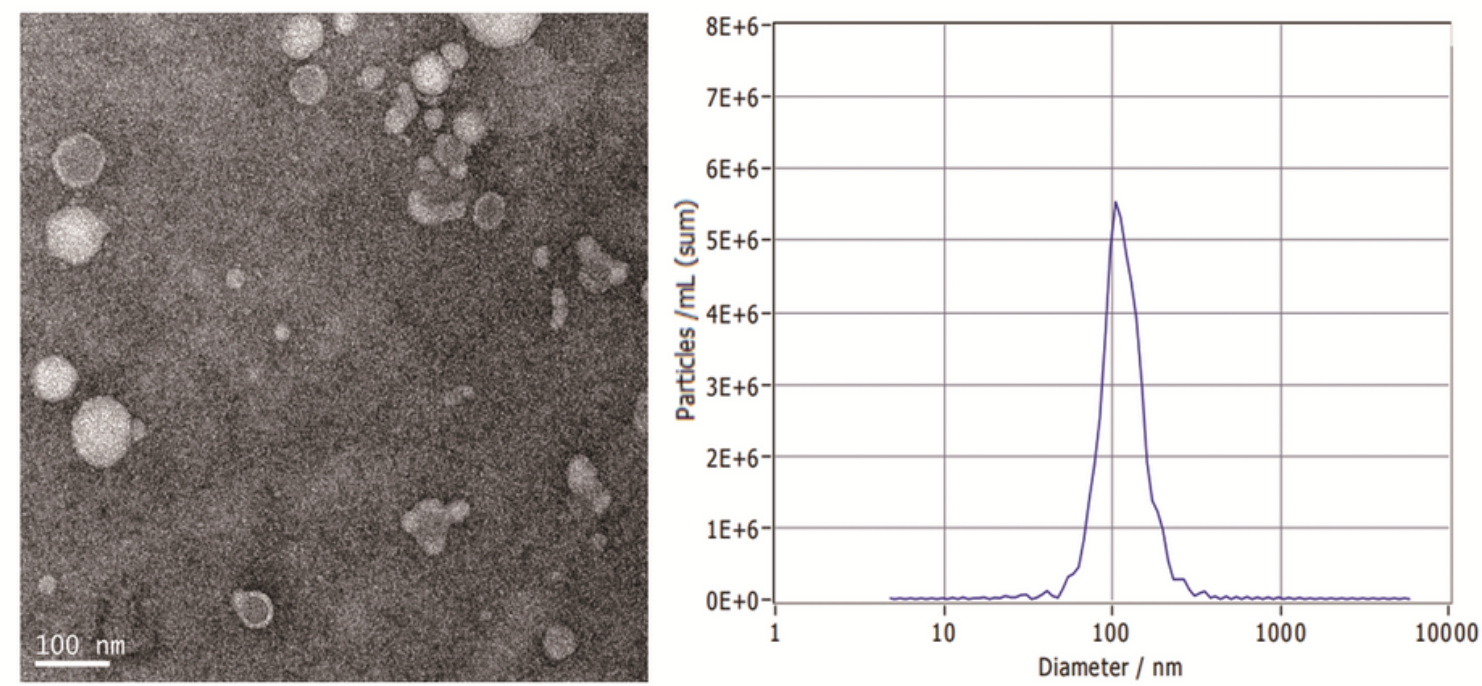

CD9

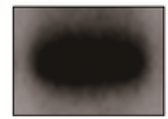

CD63

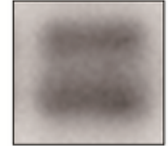

TSG101

Annexin V

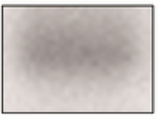

Figure 2

Patient exosome characterization 
A

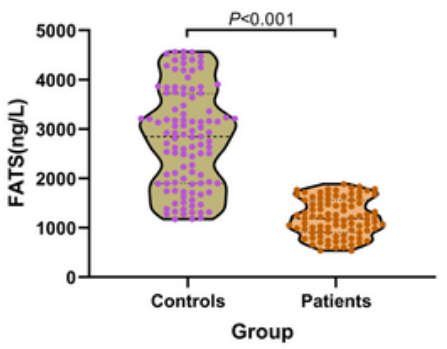

C

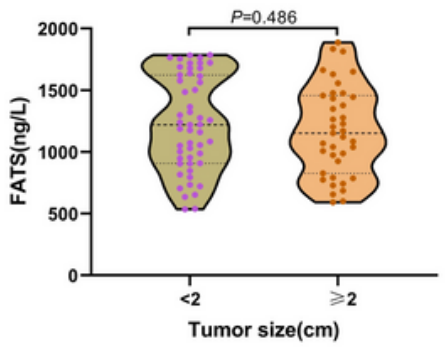

E

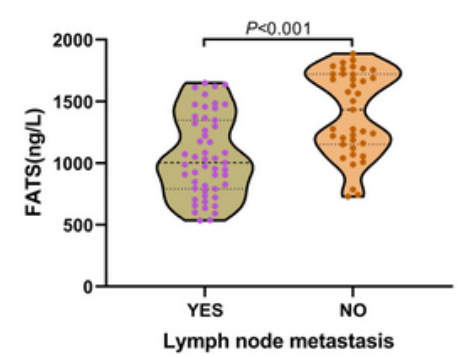

G

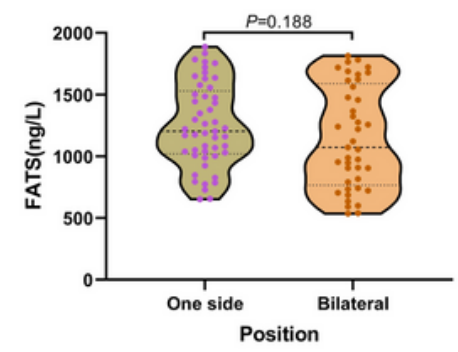

I

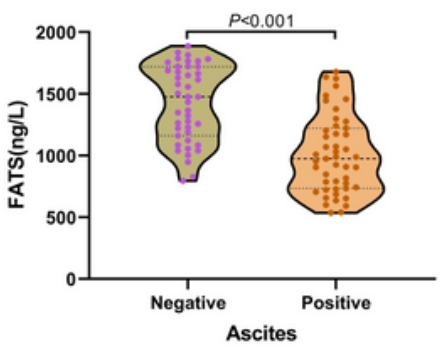

B

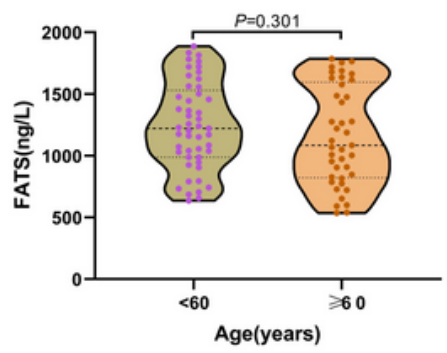

D

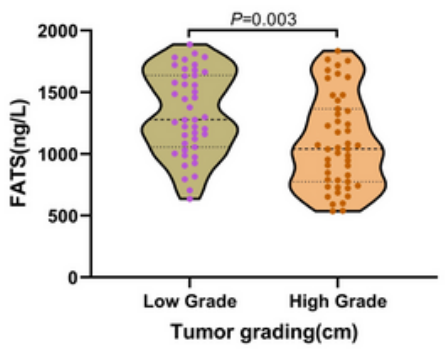

$\mathrm{F}$

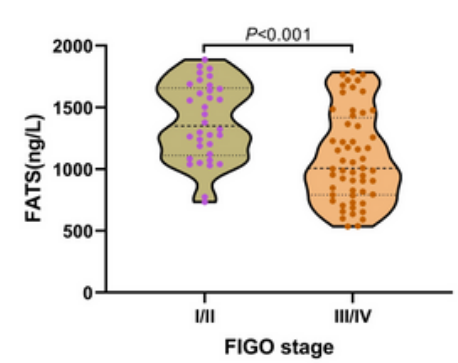

$\mathrm{H}$

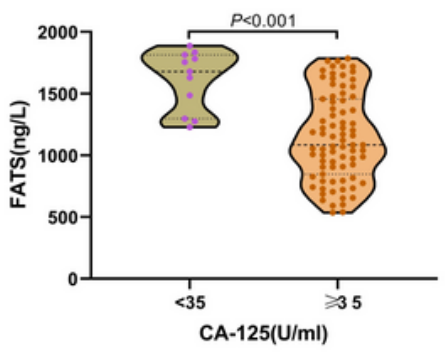

\section{Figure 3}

The levels of plasma exosome-derived FATS in OC patients 
A

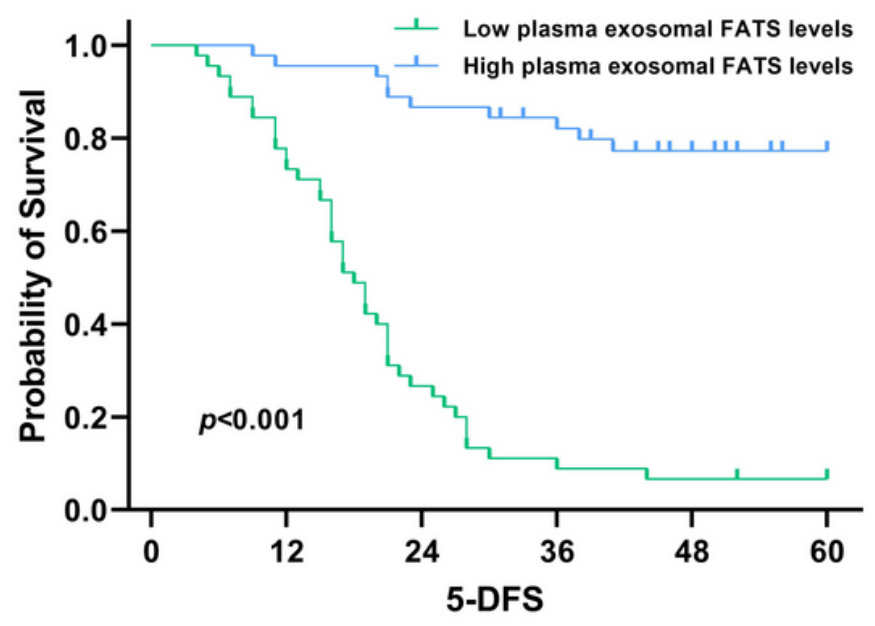

B

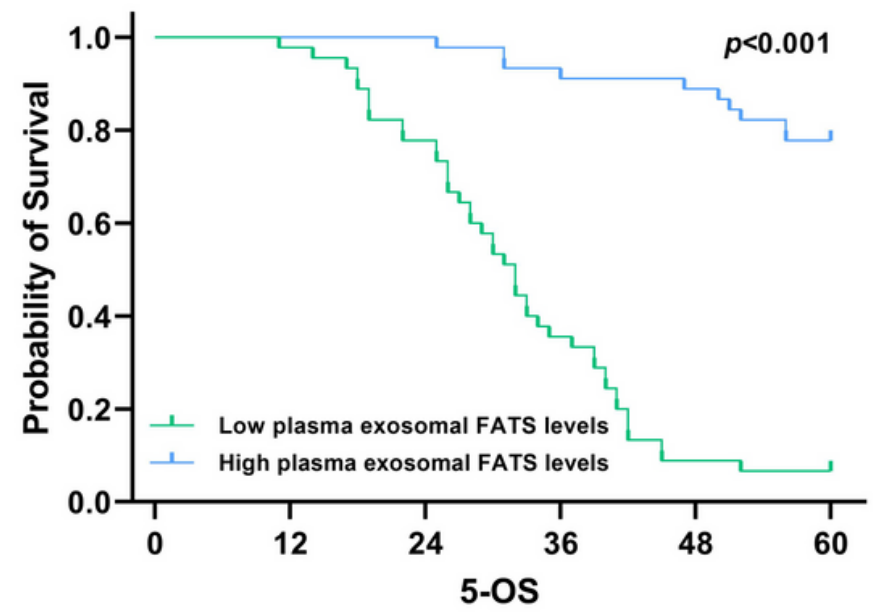

Figure 4

Association of plasma exosome-derived FATS levels with 5-DFS and 5-OS in OC patients

\section{Supplementary Files}

This is a list of supplementary files associated with this preprint. Click to download.

- uneditedversionsoftheWesternblots.pdf 